\title{
Possibilities of Personal Health Status Monitoring
}

\author{
Jan Havlík ${ }^{1}$, Jan Dvorák ${ }^{1}$, Jakub Parák ${ }^{1}$, Matouš Pokorný ${ }^{1}$, Lenka Lhotská ${ }^{1}$, Petr Panýrek ${ }^{2}$ \\ ${ }^{1}$ Faculty of Electrical Engineering, Czech Technical University in Prague, Czech Republic \\ ${ }^{2}$ HIGH TECH PARK, Prague, Czech Republic
}

\begin{abstract}
Background: There are a lot of possibilities how to monitor the patient using wireless technologies and how to initiate the appropriate action in life-threatening situations. The paper focuses on those issues. The overview of basic principles and outline of significant advantages and disadvantages of the methods are presented.

Objectives: The main aim of these solutions is to ensure safety of the elderly and impaired people in their everyday life, and especially, to enable the possibility to stay in their natural environment (home, family, etc.) instead of institutionalizing them.

Methods: Two solutions for monitoring and providing better life quality conditions are presented in more detail. The specific solution for monitoring vital signs and classifying urgent states using telemedical system - the Intelligent Primer Nurse application - is presented in detail. The smart system combining the telemedical approach and home TV computer - Home Brain system - is described.
\end{abstract}

\section{Correspondence to:}

\section{Jan Havlík}

Department of Circuit Theory, Faculty of Electrical Engineering,

Czech Technical University in Prague

Address: Technická 2, CZ-16627 Prague 6

E-mail: xhavlikj@fel.cvut.cz
Results: Both solutions have been tested in real use and under different conditions. The Intelligent Primer Nurse system has been tested during long term vital signs monitoring including several activities - walking, running and idle standing. The Home Brain system has been evaluated during a pilot study with a group of elderly in their real life. The user experiences have been investigated by a moderated discussion.

Conclusions: Finally, we discuss the future development in this domain.

\section{Keywords}

Telemonitoring, telemedicine, vital signs monitoring, assistive technologies

\section{Introduction}

Currently, there is an increasing demand for smart solutions helping and serving in daily life. Teenagers, students, young people, and also many people in productive age are the users of smart phones, tablets, netbooks, and other smart and portable devices. Although elderly people often mistrust these devices, it could be observed during the last years that there is an increasing interest in these solutions also in post-productive age. Elderly people become suddenly aware that the smart solutions could significantly help them managing their daily life.

Simultaneously with the decrease of mobile device prices, the number of mobile applications has been growing. Especially in the field of personal health state monitoring, the speed of development of applications is very rapid. Actually we can measure many physiological parameters from a human body in relatively easy way: elec-
EJBI 2014; 10(1):11-14

received: December 17, 2013

accepted: February 9, 2014

published: February 28, 2014 trocardiogram (ECG), heart rate $(\mathrm{HR})$, breathing rate, body temperature, blood pressure, etc. [1. Many clinical trials have been performed, e.g. [2, 3] assessing the usefulness and efficiency of telemonitoring systems.

Many of the existing solutions could be easily adopted and adapted to elderly and applied in real life. The main aim of almost all of these applications is to safeguard the elderly and impaired people in their daily life, and more specifically to enable them stay even longer in their own natural environment (home, family, etc.) rather than being institutionalized.

Based on the facts mentioned above, one of the most actual problem is to design and develop the smart solutions, embedded devices, and telemonitoring applications as user-friendly as possible 4, 5]. The paper presents two examples of solutions for monitoring health status and providing better life for elderly or impaired persons. 


\subsection{Methods}

The first solution is a specific solution for monitoring vital signs and classifying urgent states using telemedical system - the so-called Intelligent Primer Nurse application. The second one is a smart system combining the telemedical approach and home TV computer - the socalled the Home Brain system.

\subsection{Intelligent Primer Nurse}

The Intelligent Primer Nurse is a device for a continuous monitoring of vital signs that acts as a personal and portable vital signs monitor (see the Fig. 1 for the laboratory realization). The device is able to activate alarms in case the vital signs are not any longer in the specified, pre-defined range. Chronically diseased, elderly, and other threatened persons including persons at risk for a stroke are the target group of users.

The device as such is based on the EvoPrimer [6] development kit. The basic kit is supplemented with the extension board, the input module, and communication modules. The device allows measuring electrocardiography (ECG), plethysmography (PPG) and activity (accelerometry) signals, displaying the values of the signals on the screen in real time, and processing these signals accordingly.
The alarm is activated whenever the heart rate is out of the pre-defined range or when the user is inactive for long time. The activity alarm could be inhibited by pushing the activity button. The device is able to send alarm signal wirelessly to the subordinate PC system or directly to the control center.

\subsection{Home Brain}

Home Brain is a unique system put into practice at the end of 2011 after more than five year of advanced research. The system is technically designed as a small device like a set-top-box connected to a standard TV set (see Fig. 2). The main aim of the device is to provide the gate functionality to the Internet, multimedia services, senior monitoring, health state monitoring, social networking, remote control of home devices, intelligent security system, etc. The Home Brain system allows networking without demand for complicated control in the easiest way possible. This approach significantly increases the ability of elderly to stay at their own home environment instead of moving them into centers of institutionalized care such as senior houses [7].

\section{Results}

Both systems presented above have been tested in real use and under different conditions.

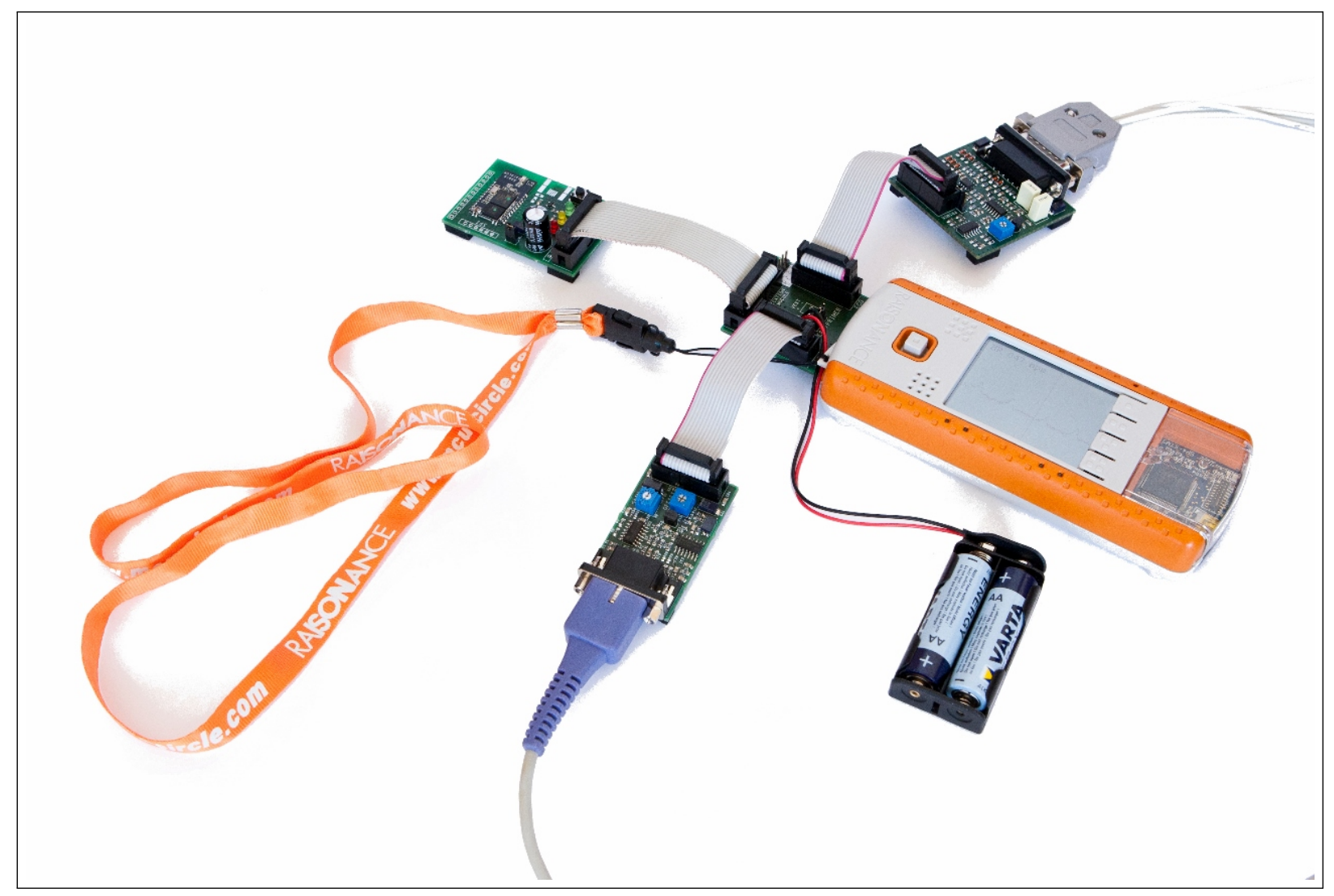

Figure 1: Intelligent Primer Nurse. 
The Intelligent Primer Nurse application has been evaluated in simulated situations, the so-called test scenarios. The system has been tested during long term vital signs monitoring including several activities - walking, running and idle standing. The reaction to lifethreatening situations like low heart rate, no activity (for example after the falling down) etc. has been also tested [8].

The Home Brain system has been evaluated during a pilot study with a group of elderly in their real life. During this study the Home Brain has been tested by 5 users, 4 women and 1 man, from 66 to 78 years old. The user experiences have been investigated by a moderated discussion. As a main advantage of the system the users high-lighted the simplicity and the intuitiveness of system control. The respondents had no manuals, they learned only by using the system. They agree that the most important issue is not to be afraid of using the Home Brain. As the most frequently used functions they activate watching TV, using a TV archive (instead video-recorder), listening to radio, Skype calling, instant messaging, using the photo archive, managing details about their home (payments, important decisions, dates of medical visits etc.) and evidence of health status. The respondents have been also asked for arguments for potential new users. As crucial arguments they mention the simplicity of the use, the comfortableness, and the enhancement of communication possibilities [9].

Recently, the Home Brain has been introduced to the market.

\section{Conclusion}

There are many possibilities to help elderly and impaired persons in their everyday life. Using the up-to-date techniques and applications such as smart phones, tablets and specialized portable devices, it is relatively cheap and easy to monitor the health status continuously, to send a message in case the health status is not all right, and to additionally network them with their family, friends, or caregivers. For further development we have to keep in mind the requirement on simple and intuitive control of these devices that can increase their acceptance in population. Another issue that is not yet satisfactorily solved is the interoperability of different solutions. The ultimate

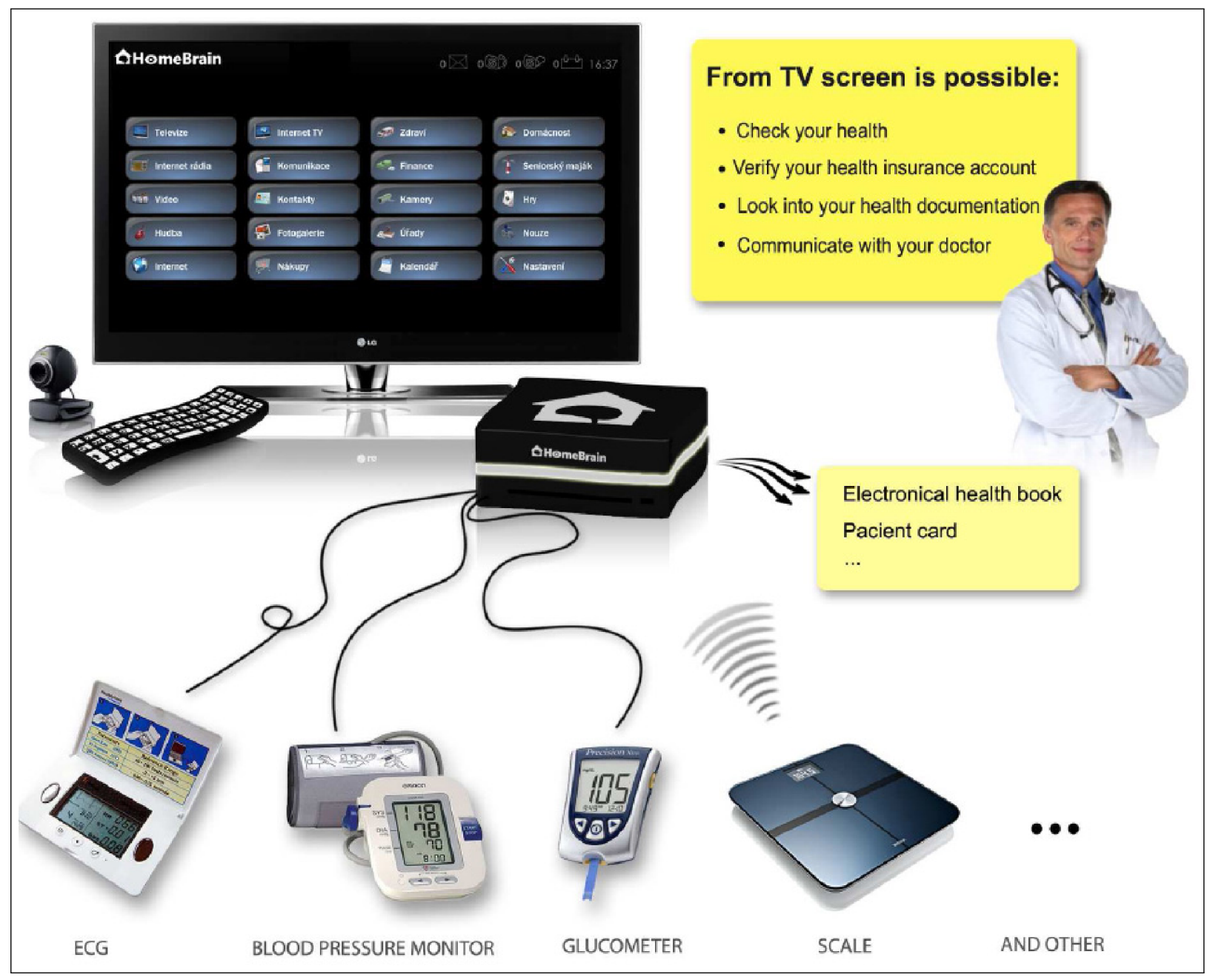

Figure 2: Home Brain. 
goal is to introduce the "plug-and-play" concept for easier extension of the applications.

The number of possibilities to monitor personal health status is increasing as fast as the prices of new technologies are getting down.

\section{Acknowledgements}

This work has been jointly supported by the grant No. G3 902/2013 of the University Development Foundation, by the grants No. SGS12/143/OHK3/2T/13 and SGS13/203/OHK3/3T/13 of the Czech Technical University in Prague, and by the research program No. MSM 6840770012 of the Czech Technical University in Prague (sponsored by the Ministry of Education, Youth and Sports of the Czech Republic).

\section{References}

[1] Xiao-Fei T., Yuan-Ting Z., Poon C., Bonato P.: Wearable medical systems for p-health. IEEE Reviews in Biomedical Engineering $2008 ; 1: 62-74$

[2] Martin-Lesende I., Orruño E., Cairo C., Bilbao A., Asua J., Romo M., Vergara I., Bayn J., Abad R., Reviriego E., Larrañaga J.: Assessment of a primary carebased telemonitoring intervention for home care patients with heart failure and chronic lung disease. The telbil study. BMC Health Services Research 2011, 11(56).

[3] Kraai I., Luttik M., de Jong R., Jaarsma T., Hillege H.: Heart failure patients monitored with telemedicine: Patient satisfaction, a review of the literature. Journal of Cardiac Failure 2011, 17(8):684-690

[4] Chan M., Esteve D., Escriba C., Campo E.: A review of smart homes - present state and future challenges. Computer methods and programs in biomedicine 2008, 91(1):55-81.

[5] Lhotská L., ŠtěpánkovJ. O., Pěchouček M., Šimák B., Chod J. ICT and eHealth projects. Telecom World (ITU WT), Technical Symposium at ITU, Piscataway: IEEE; 2011; 57-62.

[6] EvoPrimer for STM32F103VE. STM [Internet]. 2012 [cited 2012 Feb 15]. Available from: http://www.stm32circle.com/resources/stm32Eprimer.php.

[7] HIGH TECH PARK: Project3B HomeBrain. High Tech Park [Internet]. 2011 [cited 2012 Feb 15]. Available from: http://www.htpark.eu/en/solutions/r1-project3bhomebrain/.

[8] Parák J., Dvořák J., Havlík J.: Device for long term measurement of heart rate. ACM Digital Library: Proceedings of 4th International Symposium on Applied Sciences in Biomedical and Communication Technologies [CD-ROM], New York: ACM, 2011, 1-5.

[9] Příbová, M.: HOMEBRAIN Opinions and feelings of users about multifuncional device (in Czech). Unpublished research note, ILOL, 2010. 